\title{
Ciencias básicas en Obstetricia y Ginecología: una necesidad
}

Una de las manifestaciones cada vez más recurrentes de los estudiantes de medicina y de otras carreras del área biomédica en Chile, es su creciente interés por ser partícipes directos de medios que les permita embeberse de conocimiento directamente en el desarrollo de actividades de investigación, en laboratorios de investigación profesionales.

Este fenómeno, resulta en primera instancia de su propia insaciable inquietud intelectual, en etapas tanto iniciales como avanzadas y terminales de sus carreras. Sumado a esto, un papel cada vez más determinante en el despertar de esta iniciativa en estos futuros profesionales, surge de la información y formación que sus profesores o tutores 'puedan' o 'estén habilitados' (i.e., que cuenten con el conocimiento necesario) para brindarles.

Para enseñar no solo es necesario contar con el deseo y la posibilidad de hacerlo, sino que se debe contar además con un grado de conocimiento, el más apropiado posible, que permita entregar información certera en un área determinada del conocimiento. Así, en un movimiento que en países desarrollados presenta un frente de avanzada significativamente mayor, comparado con nuestra realidad regional y local, los profesionales clínicos que desarrollan una activa y fructífera docencia han requerido en algún momento de su carrera académica el formarse a un nivel avanzado y de frontera en las áreas de desarrollo que les corresponde.

En Chile y la Región, la realidad es diferente. En general, un número reducido de profesionales médicos recién egresados, deciden tomar la también aun escasa oferta de formación de postgrado a nivel de doctorado o magíster en ciencias médicas (medicina en todas sus áreas de especialización) o biomédicas (ciencias médicas con un fuerte componente de ciencias básicas).

Aún cuando la formación de pregrado en medicina es sólida y de alta exigencia, sin espacio a mayor cuestión, los profesionales egresados han tenido una escasa oferta de oportunidades para desarrollarse en términos de potenciar sus conocimientos de medicina, con otras herramientas que les permita conocer en profundidad mecanismos específicos (sistémicos, celulares y moleculares) que ocurren a nivel local (ej., eventos inmediatos y adaptativos que se gatillan en una herida), frente a alteraciones sistémicas (ej., los mecanismos de adaptación cardiovascular a la hipoxia), alteraciones tisulares (ej., los mecanismos de regeneración tisular) o celulares (ej., la dinámica de los procesos de angiogénesis bajo el control del endotelio).

Hoy, con el avance de las ciencias básicas, finamente también denominada 'ciencia fundamental', muchos de estos aspectos son abordados en profundidad en laboratorios de investigación profesionales con especialistas que no provienen necesariamente de una formación en el área médica, sino cercano a su totalidad (80-90\%) corresponden a profesionales del área investigativa por naturaleza, tales como bioquímicos, biólogos, tecnólogos y otros de formación afín (1).

Sin embargo, cada vez es mayor el interés y el acceso a recursos técnicos (equipamiento, protocolos prácticos, nuevos reactivos) para investigación en el área clínica, lo que resulta en una mejor y rápida comprensión de los resultados publicados por ejemplo en estudios clínicos retrospectivos. Así también lo anterior se ve en el aumento de oportunidades para el uso de herramientas lógicas y de estrategia experimental, fundamentada en el conocimiento dado por la propia formación profesional, la información clínica disponible y el conocimiento acumulado de fenómenos celulares y moleculares específicos, enriqueciendo la oportunidad de propuestas de estudios prospectivos. Ciertamente, nada de esto resulta ser de fácil logro y desarrollable en condiciones naturales, sino que en efecto necesita de ayuda dada en paralelo, que le permita al individuo realizar sus inquietudes con demostraciones experimentales directas o concretas en un 
laboratorio. Así, surge entonces, en los tiempos actuales, la necesidad de desarrollar investigación fundamental en las áreas del conocimiento de la medicina en general y especializada.

Es interesante notar que las investigaciones en el área de Obstetricia y Ginecología han aumentado exponencialmente desde los años 70 a la fecha. La información disponible en bases de datos abiertas (PubMed-NCBI) muestra un fuerte aumento en el interés por publicar en temas específicos relacionados con todos los aspectos posibles, directos e indirectos, de la fisiología y fisiopatología de la placenta como un órgano esencial en el desarrollo del ser humano desde su concepción (Figura 1). Igualmente, este fenómeno se repite en relación a estudios referidos a la fisiología fetal en la salud y la enfermedad. En estas investigaciones, a pesar de seguir siendo primariamente dirigidas (i.e., investigador principal o responsable del proyecto) por investigadores básicos, un número creciente de investigadores del área clínica hoy son líderes de grupos de investigación.

En países con un consolidado y longevo desarrollo en investigación en Obstetricia y Ginecología (ej. Norteamérica y diversos países europeos) un número significativo de los proyectos en el área, son hoy dirigidos por investigadores principales cuya formación inicial es en clínica, estudios que son desarrollados en coautoría con investigadores en ciencias básicas. Nuestra realidad en Chile es, sin embargo, limitada a grupos de especialistas en Obstetricia y Ginecología con proyectos de investigación financiados por las instituciones en donde desarrollan su actividad profesional o por la adjudicación de fondos extramurales en Chile (CONICYT,

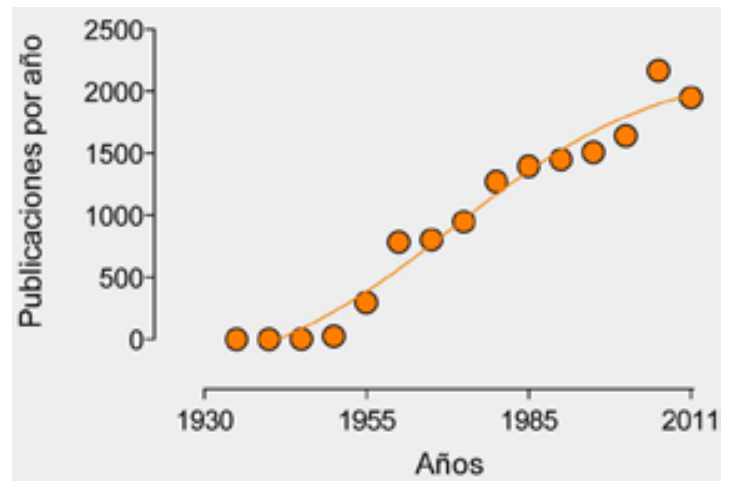

Figura 1. Publicaciones en obstetricia y ginecología. Las cifras corresponden al número de publicaciones ISI por año indexadas en la base de datos PubMed.org bajo los términos 'human + placenta' entre los años 1930 a Diciembre 31 del 2011.
FONDEF, FONIS, entre otros) y en el extranjero (NIH-USA, Comunidad Europea, AECID-España, entre otros). Esto es, aunque limitado, muy alentador, pues estos grupos cuentan con uno o dos líderes con vasta experiencia clínica que complementan finamente su propio conocimiento, con las habilidades y experiencias de investigadores profesionales en ciencias básicas.

En la actualidad las publicaciones de grupos chilenos en el área de Obstetricia y Ginecología son el resultado exquisito de la complementariedad de tecnología y conocimiento, antes que una prestación de servicio profesional de parte de los investigadores en ciencias básicas. La experiencia internacional en el área muestra y obliga a desarrollar investigación altamente competitiva con un claro y definitivo componente en ciencias básicas. Solo esta opción permite aproximarse a una caracterización más clara y precisa de dianas o blancos terapéuticos para el tratamiento de enfermedades del embarazo de alta incidencia en nuestro país (ej. diabetes gestacional, preeclampsia, restricción de crecimiento intrauterino, entre otras) $(2,3,4)$.

La investigación en Obstetricia y Ginecología ha sido un tema profundamente olvidado en términos de la búsqueda del conocimiento inherente a la caracterización de mecanismos específicos que determinen o resulten de patologías del embarazo. Esto es un camino que necesariamente debería complementar la serie de factores de riesgo reconocidos (en algunos casos muy bien establecidos) y sugestivos de alteraciones en el desarrollo y potencialmente viabilidad del feto en desarrollo, con los signos y síntomas manifestados por la madre en un embarazo patológico.

Con el conocimiento de mecanismos específicos acerca de las bases fundamentales de las enfermedades, las decisiones a tomar en el tratamiento de una paciente con alguna patología del embarazo deben basarse justamente en ese conocimiento, el cual debe ser integrado con aquéllos síntomas reconocidos en la paciente por el médico tratante y los datos complementarios disponibles de parte de las investigaciones en ciencia básica. Estos conocimientos sin duda deben ser considerados como parte de los elementos del diagnóstico médico y decisiones de tratamiento a la madre y el feto en desarrollo.

Un ejemplo preciso surge de las investigaciones que establecen que pacientes que desarrollan preeclampsia durante su embarazo, presentan niveles plasmáticos elevados de adenosina, un nucleósido endógeno que actúa como un potente vasodilatador en la mayoría de los lechos vasculares (5), antes de las primeras manifestaciones de la enfermedad 
(6). Recientemente se ha propuesto que este aumento en el nivel plasmático de adenosina podría constituir una primera señal indicativa de disfunción endotelial asociada a esta patología (7). Otras investigaciones demuestran que adenosina también se encuentra elevada en la sangre umbilical al momento del nacimiento en embarazos que han cursado con diabetes gestacional (8). Esta observación complementa lo informado tempranamente en mujeres que se embarazan y que tienen un diagnóstico previo de diabetes mellitus (i.e., diabetes pregestacional) o en mujeres diagnosticadas con diabetes gestacional donde los niveles plasmáticos de adenosina en la madre son mayores a los detectados en mujeres que cursaron con embarazos no patológicos (9). Así, de esta manera absolutamente tangible, la ciencia básica está permanentemente exaltando al quehacer médico en el área que las decisiones deban ser tomadas en base al conocimiento de todo el conjunto de información.

Actualmente, la relación de un alumno de postgrado en la especialidad de Obstetricia y Ginecología con la ciencia básica es mínima. Se considera que esta actividad es consumidora de tiempo, aquél que los alumnos normalmente no tienen, o que lo tienen escasamente protegido para esta actividad en la curricula en Chile. Aún peor, se ha considerado incluso poco determinante, pues constituye una actividad cuyos resultados no tendrían una aplicación inmediata (i.e., en el momento de diagnosticar la enfermedad) a la resolución de una situación adversa durante el embarazo de una mujer. La prisa actual evidenciada en esta actitud de sentir la necesidad de proceder inmediatamente para intervenir lo más mínimamente al paciente con el fin de solucionar o mitigar en parte las complicaciones de su enfermedad, lo cual se ajusta ciertamente a las funciones inherentes del médico, raya en la línea de subenteder o mal valorizar la necesidad de proyectar el conocimiento generado hoy hacia al futuro, de la intervención en pacientes con enfermedades del embarazo. No existe una relación entre ciencia básica y aplicación que sea inmediata, pues todos los elementos y fases en la aprobación de un protocolo terapéutico requieren de ensayos en diferentes etapas de complejidad. Así, la ciencia básica en Obstetricia y Ginecología deberá irremediablemente seguir este camino para asegurar la buenaventura de un futuro procedimiento, para el tratamiento de la patología y una mejor calidad de vida de la mujer, el niño y su entorno familiar inmediato.

La formación de postgrado en Obstetricia y Ginecología en Chile, cuenta con algunas ventanas que son esperanzadoras en cuanto a requerir a alumnos de postgrado en esta especialidad que desarrollen actividades de ciencias básicas complementarias en su formación curricular. Es así como alumnos de pregrado que han tenido la posibilidad de realizar investigación básica en laboratorios abocados al área, consideren la posibilidad de especializarse en Obstetricia y Ginecología. Aun más, un número no despreciable de médicos recién egresados han optado en los últimos años por desarrollar programas de postgrado de doctorado y magíster en el área. Los temas centrales de los trabajos de tesis, una actividad que es requisito obligatorio de cumplir en estos programas de postgrado, son casi en su totalidad en temas clínicos cuyo componente de ciencia básica es esencial, ocupando $\sim 75 \%$ o más del trabajo realizado para alcanzar conclusiones. Estos estudios han sido altamente competitivos, publicados en revistas de la especialidad, y han permitido una visión amplia y con conocimiento de los problemas obstétricos y ginecológicos a quienes lo han desarrollado.

No existe la opción en la actualidad de permanecer como especialista en Obstetricia y Ginecología sin una formación de avanzada en ciencias básicas, pues el desarrollo del área en el contexto internacional así lo requiere. Solo contando con estas herramientas se podrá avanzar en el conocimiento de las bases intrínsecas de las enfermedades del embarazo que afectan tanto a la madre como al niño que está por nacer. Sumado a este conocimiento de la enfermedad misma en el embarazo, debemos estar conscientes que las alteraciones en el desarrollo fetal en este tipo de patologías tienen una consecuencia claramente documentada en el desarrollo de enfermedades en la vida adulta, tales como hipertensión, diabetes gestacional y diabetes mellitus tipos 1 y 2 , obesidad, resistencia a insulina, entre otras (10). De esta manera, la incorporación de conocimientos vivenciales y no solo teóricos, al curriculum en alumnos de postgrado en Obstetricia y Ginecología de nuestro país es crítico. De ser posible incorporar estas herramientas en la formación de estos especialistas, será clave y determinante en su formación profesional, y en el futuro de las generaciones que vienen en esta área de la medicina prontamente, esperamos, investigativa y de frontera. Un mayor conocimiento de las bases intrínsecas de las enfermedades en el área (i.e., mecanismos celulares y moleculares con redundancia en alteraciones sistémicas en la madre y el niño en desarrollo), permitirá enfrentar la patología con herramientas precisas cuya finalidad sea proteger a la madre y al niño en desarrollo en nuestra población. Así, y solo así, la educación de las nuevas generaciones en Obstetricia y Ginecología tendrán el sustento ético y profesional que se 
requiere para avanzar a la par con el conocimiento, no solo de aquél encontrado en las publicaciones científicas, sino también con aquel conocimiento generado por la agudeza intelectual e investigativa de profesionales del área.

Dr. Luis Sobrevía L. MSc, PhD. Profesor Asociado.

Departamento de Obstetricia y Ginecología. Laboratorio de Fisiología Celular y Molecular (CMPL).

Facultad de Medicina. Pontificia Universidad Católica de Chile.

\section{REFERENCIAS}

1. Fondo Nacional de Desarrollo Científico y Tecnológico (FONDECYT). (2012) Sección Estadísticas y Productividad. URL: http://www.fondecyt.cl/578/propertyvalue-57546.html. 2012.

2. Belmar C, Salinas P, Becker J, Abarzúa F, Olmos $\mathrm{P}$, González $\mathrm{P}$, Oyarzún E. Incidencia de diabetes gestacional según distintos métodos diagnósticos y sus implicancias clínicas. Rev Chil Obstet Ginecol 2004;69:2-7.

3. Huidobro A, Fulford A, Carrasco E. Incidence of gestational diabetes and relationship to obesity in Chilean pregnant women. Rev Med Chil 2004;132:931-8.
4. Ministerio de Salud de Chile, MINSAL. Encuesta Nacional de Salud ENS Chile, 2009-2010. 2011.

5. Eltzschig HK. Adenosine: an old drug newly discovered. Anesthesiology 2009;111:904-15.

6. Espinoza J, Espinoza AF, Power GG. High fetal plasma adenosine concentration: a role for the fetus in preeclampsia? Am J Obstet Gynecol 2011;205:485. e24-e27.

7. Escudero C, Casanello P, Sobrevia L. Human equilibrative nucleoside transporters 1 and 2 may be differentially modulated by $\mathrm{A} 2 \mathrm{~B}$ adenosine receptors in placenta microvascular endothelial cells from preeclampsia. Placenta 2008;29:816-825.

8. Westermeier F, Salomón C, González M, Puebla C, Guzmán-Gutiérrez E, Cifuentes F, Leiva A, Casanello P, Sobrevia L. Insulin restores gestational diabetes mellitus-reduced adenosine transport involving differential expression of insulin receptor isoforms in human umbilical vein endothelium. Diabetes 2011;60:1677-87.

9. Maguire MH, Szabo I, Valko IE, Finley BE, Bennett TL. Simultaneous measurement of adenosine and hypoxanthine in human umbilical cord plasma using reversed-phase high-performance liquid chromatography with photodiode-array detection and on-line validation of peak purity. J Chromatogr B Biomed Sci Appl 1998;707:33-41.

10. Gluckman PD, Hanson MA. Living with the past: evolution, development, and patterns of disease. Science 2004;305:1733-6. 